\title{
Entre l'État, les institutions et les sujets: Considérations sur l'assujettissement, la résistance et les moralités
}

Entre o Estado, as instituições e os sujeitos: Considerações sobre o assujeitamento, a resistência e as moralidades

Between the State, the institutions and the subjects: Considerations on the subjectification, the resistance and the moralities

\section{Theophilos Rifiotis}

\section{(2) OpenEdition \\ Journals}

Édition électronique

URL : http://journals.openedition.org/aa/3339

DOI : $10.4000 /$ aa.3339

ISSN : 2357-738X

\section{Éditeur}

Programa de Pós-Graduação em Antropologia Social (UnB)

Édition imprimée

Date de publication : 1 décembre 2018

Pagination : $337-359$

ISSN : 0102-4302

Référence électronique

Theophilos Rifiotis, «Entre l'État, les institutions et les sujets: Considérations sur l'assujettissement, la résistance et les moralités », Anuário Antropológico [En ligne], v.43 n.2 | 2018, mis en ligne le 26 mai 2019, consulté le 27 avril 2021. URL : http://journals.openedition.org/aa/3339 ; DOI : https://doi.org/ 10.4000/aa.3339

\section{(c) (i) (9)}

Anuário Antropológico is licensed under a Creative Commons Atribuição-Uso Não-Comercial-Proibição de realização de Obras Derivadas 4.0 International. 


\section{Entre l'État, les institutions et les sujets: Considérations sur l'assujettissement, la résistance et les moralités}

Theophilos Rifiotis

UFSC

Ce n'est donc pas le pouvoir, mais le sujet qui constitue

le thème général de mes recherches.

M. Foucault, Dits et écrits

Ce texte vient d'une présentation faite dans le cadre du groupe de travail "Rethinking the concept of moral economy: anthropological perspectives" au Congrès de l'EASA (European Association of Social Anthropologists) à Milan en 2017. ${ }^{1}$ Face à la pluralité conceptuelle de la notion d'économie morale, il faut discuter son potentiel heuristique et sa portée théorique pour le développement d'une anthropologie critique, ou d'une anthropologie morale, comme l'a très bien défini Didier Fassin (2008).

La notion d'économie morale est devenue centrale dans mes recherches et mon enseignement. Cette notion clé m'a offert de nouvelles possibilités analytiques du problème théorique qui me préoccupe: la violence. Particulièrement, la judiciarisation de la violence du genre et la place du sujet qui sont des questions issues de longues années de recherches de terrain et de directions de thèses, que je vais tâcher d'expliquer.

Quant à l'importance du sujet pour les études des économies morales, je commencerai en situant mon parcours de recherche par rapport à la morale et à la question du sujet, et les raisons qui m'ont poussées à discuter les possibilités qu'elles offrent à la théorie des économies morales. ${ }^{2}$ Ensuite, pour aller plus en avant dans le dialogue avec les économies morales, le texte prendra la forme d'une exégèse, disons dialogique de l'Introduction du livre "Juger, réprimer, accompagner. Essai sur la morale de l'État” (Fassin et al., 2013), ce qui me permettra de discuter les fondamentaux de la praxis, tel que je l'envisage dans ce texte et leurs rapports avec une approche par la notion de sujet. De telle sorte que cette Introduction sera 
à la fois mon point de départ et mon matériau de réflexion. En fait, le choix de cette Introduction n'est donc pas dû au hasard, bien au contraire, il représente en quelque sorte une évaluation de la place que cet ouvrage occupe dans le champ des études de l'économie morale, ainsi que sa portée théorique sur les questions de politiques sociales, au centre de mes enquêtes actuelles sur les droits humains et la judiciarisation de la violence de genre (Rifiotis, 2014a, 2015).

Disons d'emblée qu'il ne s'agit nullement d'une critique du texte, car c'est ce même texte qui m'a inspiré et m'a donné les jalons pour poursuivre ma démarche analytique et ainsi, mieux cerner mes inquiétudes théoriques. L'ouvrage est avant tout un grand effort collectif pour trouver un langage qui puisse "traduire" ou mieux encore articuler l'agenda social avec les questions académiques, dans un langage approprié au grand public, sans perdre la rigueur. ${ }^{3}$ De telle sorte que "Juger, réprimer, accompagner" soit un ouvrage de la plus grande importance pour les études des services publics et le développement des politiques sociales. Ce qui en fait d'ailleurs, son atout majeur: un exercice concret d'anthropologie morale.

Avant de développer mon argumentation, je voudrais dire avec insistance que les questions soulevées par cette Introduction s'inscrivent dans la perspective d'une anthropologie morale (Fassin, 2008) et qu'elles nous posent des questions théoriques, lesquelles nous invitent (voire convoquent) à penser aux effets qu'elles peuvent avoir dans le débat public. Je fais alors miennes les propositions de la Conclusion de “Juger, réprimer, accompagner" et j' espère en tirer des conséquences cohérentes avec cette posture pour le débat que je propose sur le sujet et les économies morales:

Il faut se faire conséquentialiste, autrement dit, il faut penser les conséquences de ce que font l'État et ses agents, notamment dans deux domaines que le philosophe de la biopolitique a remarquablement ignorés: l'injustice et l'inégalité. Au terme de notre voyage au cour de l'État, il nous semble que c'est à ces deux critères, aux frontières de la morale et de la politique, qu'il faut juger les sociétés contemporaines. (Fassin et al., 2013: 356)

\section{Remarques préliminaires}

Un premier point à expliquer pour bien situer mon propos, c'est de rappeler le début de mes recherches dans le domaine de l'ethnologie africaine. Je me suis alors consacré à l'étude des rapports entre savoir et pouvoirs chez les peuples dits "bantous", en Afrique Centrale et Australe, particulièrement chez les Makonde du 
Mozambique. Mes études des récits traditionnels sur l'image du vieillard m'ont permis d'analyser la place structurante des conflits entre les groupes d'âge (2007a). Ils sont à l'origine de mes interrogations sur les notions de "violence" et de "conflit" telles qu' elles étaient présentes dans les débats publics au Brésil aussi bien que dans la littérature spécialisée. Pour une vue d'ensemble, il faut voir le recensement fait par Alba Zaluar (1999), mais aussi la littérature classique dans ce domaine. Pour ne donner que quelques références particulièrement liées à la productivité de "violence" (Rifiotis, 2008a), on rappelle les contributions fondamentales de George Simmel dans son essai sur le conflit (1992), de Walter Benjamin dans "Critique of violence” (1978), de Max Gluckman et ses études sur les rituels de rébellion (1963), ainsi que celles de Pierre Clastres dans la "société contre l'état" (1988) et Jack Katz dans son ouvrage "Seductions of crime" (1988), parmi tant d'autres. À partir de ce matériau empirique, j'ai d'abord étudié les médias et la "violence policière” (1999) et ensuite, les procès pénaux de cas de parricide (2011b), puis j'ai essayé de produire une révision de la littérature sur la "violence" (1997, 1998, 1999, 2006, 2008a).

La problématisation ${ }^{4}$ de la notion de "violence" était devenue centrale dans mon parcours de recherche, centrale par son importance dans l'agenda social et académique depuis les années 1990 au Brésil. Dans les grandes lignes, au lieu d'un paradigme ou d'une pluralité de définitions, ce que j'ai pu identifier c'était un signifiant vide, une détermination symbolique du signifiant (Rifiotis, 1999) et un profond enracinement moral et politique de la notion de "violence". En d'autres termes, un "problème social” qui n'avait toujours pas atteint sa transformation en objet analytique et dont la grammaire qui organise son champ sémantique aurait une triple matrice: la négativité, l'homogénéité et l'extériorité. Sans pouvoir revenir sur les détails de mon approche, je voudrais rappeler ici que la "violence" est une catégorie à la fois descriptive et qualificative fondée sur une négation, un refus, une évaluation morale des faits. Luís Roberto Cardoso de Oliveira montre très bien dans son article intitulé "Existe violência sem agressão moral?" ("Existe-t-il de violence sans agression morale?”) que la moralité est un élément fondamental pour la construction sociale de la "violence" (Cardoso de Oliveira, 2008).

En fait, il s'agit de questions posées à travers l'approche anthropologique de la violence. La question de la morale était donc déjà au cœur de mes recherches comme un horizon que j'ai pu, seulement quelques années plus tard rapprocher de la voie ouverte par les études des économies morales. En fait, je me suis consacré 
à la problématisation de la "violence" dans plusieurs travaux (Rifiotis, 1997, 1998, 1999, 2006, 2008a, 2008b, 2015), mais c'est uniquement à partir de la notion d'économies morales que les rapports entre "violence" et moralité sont devenus un incontournable centre analytique, un point d'inflexion dans mon parcours de recherche. Mes lectures des travaux de Didier Fassin, ainsi que les séminaires qu'il a présentés à l'École des Hautes Études de Paris (2016 et 2017), m’ont permis d'approfondir ma compréhension des possibilités des économies morales en tant que "concept en devenir" (Fassin, 2009).

Ces dernières années, je me suis consacré aux études sur la place de la justice et des droits humains dans les politiques sociales au Brésil (Rifiotis, 2011a, 2007b, 2014b, 2017), tout particulièrement sur la judiciarisation de la violence de genre (Rifiotis, 2004, 2008b, 2015). Il s'agit d'un mouvement plus large dans l'anthropologie brésilienne qui pourrait être appelé "anthropologie juridique" ou "anthropologie du droit”, comme en témoigne la publication faite par l'Association Brésilienne d'Anthropologie" (ABA), sous la direction de Antonio Carlos de Souza Lima, dans le livre "Antropologia e direito: temas antropológicos para estudos jurídicos" (“Anthropologie et droit: thèmes anthropologiques pour les études juridiques”) (2012). Partant de ce mouvement, mes derniers travaux m’ont permis de mettre en valeur les débats sur la croissante centralité de la justice dans les politiques sociales et le passage des politiques soutenues par la défense des sujets de droit vers des politiques centrées sur la défense des droits de sujets, une inversion capitale faite par la croissance des demandes sociales tournées vers le pouvoir judiciaire, un phénomène lié à la "judiciarisation de rapports sociaux" (Rifiotis, 2004, 2007b, 2014b, 2015, 2017; Bragagnolo et al., 2015). Mes travaux de terrain ainsi que la direction de thèses et mes cours, m'ont amené à des questions qui ont une portée politique et éthique. Je pense ici, par exemple, à la polarité victime/agresseur, à l'importance de la "violence" et à la conquête des droits dans la définition des politiques publiques. En effet, il est aisé de constater que le pouvoir judiciaire devient pour les mouvements sociaux un levier de changements sociaux (Rifiotis, 2014a, 2015). Aujourd'hui, au Brésil, l'agenda social et politique tend de plus en plus vers la judiciarisation, voire la pénalisation, sans distinction de bord politique, qu'il soit de "gauche" ou de "droite". ${ }^{5}$

Le bilan de ce parcours et l'essai de le faire dans un dialogue avec la notion d'économie morale fut présenté au Congrès de l'EASA, comme indiqué au début de ce texte. Dans cette communication, j'ai voulu traduire le point d'inflexion de 
mon intérêt dans le domaine des économies morales et de l'anthropologie morale. Cette inflexion théorique m'a permis de mettre en relief la problématique de la victimisation et de l'agentivité des sujets. De fait, juste pour souligner la complexité des défis posés, je rappelle que dans un contexte ethnographique spécifique qui est celui des pratiques policières et de la cour en matière de violence de genre, il y a une controverse pour la reconnaissance de la victime, organisée via des raisons techniques de responsabilisation dont les arguments sont articulés à partir des perceptions sociales qui sont fondamentales pour la production de la justice. Comme nous l'avons observé dans nos études ethnographiques dans les commissariats de protection de la femme (Rifiotis, 2004), ainsi qu'après le changement légal avec la Loi fédérale 11340/2006 dans les cours spécialisées dans les affaires de violence de genre (Rifiotis, 2015; Bragagnolo et al., 2015), la production de la justice est toujours un résultat découlant des approches particulières de chaque commissaire ou de chaque magistrat, qui sont strictement liées aux évaluations qu'ils (commissaires ou magistrats) se font des personnes en cause. ${ }^{6}$

Ainsi, la victimisation est un véritable catalyseur de perceptions, de sensibilité, de normativité, de politiques, de protocoles d'action. Tel que le montre, le travail de D. Fassin et R. Rechtman "L'Empire du traumatisme. Enquête sur la condition de victime” (2007), pour ne citer qu'un exemple, a joué un rôle majeur dans ma problématisation de la polarité victime/agresseur. Il en est de même pour le livre "Les constructions de l'intolérable" sous la direction de Patrice Bourdelais et Didier Fassin (2005) qui m’a beaucoup aidé dans mes analyses de l'espace moral dans les pratiques judiciaires.

Ces quelques remarques initiales me permettent de situer cette inflexion comme un discours critique vis-à-vis des catégories analytiques de base de la littérature spécialisée dans l'étude de la "violence" et de la justice au Brésil, et ses rapports avec les économies morales. Deux axes d'approche se sont constitués par l'inflexion analytique que je viens de décrire: la désontologisation de l'État et les configurations des sujets dans les pratiques judiciaires. C'est donc mon intérêt sur ces deux questions qui m'a poussé à approfondir mes réflexions sur les possibilités ouvertes quant à une approche par le sujet dans le domaine des économies morales. Comme j'essaierai de le montrer par la suite, ce qui m'intéresse tout particulièrement, c'est l'articulation entre "économies morales" et "subjectivités morales" proposées par D. Fassin. 


\section{De l'État vers les institutions}

D'une manière tout à fait cohérente avec la démarche ethnographique qui est au centre de "Juger, réprimer et accompagner", son Introduction met en avant les fondamentaux des travaux qui la suivent et nous donne un aperçu critique de la notion d'État et des rapports entre l'État et les sujets tels qu'observés sur le terrain. C'est dans ce sens que je propose une réflexion sur la notion d'État et d'individu revendiquée par l'auteur. En suite, j'essaierai de montrer quelques avantages de la notion anthropologique du sujet et plus précisément celle d'agent pour un développement de l'approche anthropologique de l'économie morale.

Pour commencer, je me concentrerai sur la notion d'État telle que présentée dans cette Introduction. Comme il le souligne dès le début, ce texte s'inscrit dans la perspective de Veena Das e Deborah Poole comme un effort de “(...) nous distancer de l'image bien établie de l'État en tant que forme administrative rationalisée d'organisation politique qui s'affaiblirait ou se désarticulerait sur ses marges." (Voire "State and its margins. Comparative ethnographies". Introduction du livre qu' elles ont dirigé 2004: 3 apud Fassin et al., 2013: 15). L'État n'est donc pas un monolithe ni un assemblage de parties comme une machine ni une totalité déjà donnée. C'est un point capital et de la plus grande actualité, aussi bien sur le plan académique que sur le plan politique. D'ailleurs, dans la Conclusion de l'ouvrage il nous est rappelé encore une fois que:

L'État n'est pas un monstre froid, c'est le corrélatif d'une certaine manière de gouverner, affirme Michel Foucault dans son cours au Collège de France. Et le problème est de savoir comment se développe cette manière de gouverner, quelle est sont histoire, comment elle gagne, comment elle rétrécit, comment elle s'étend à tel domaine, comment elle invente, forme, développe de nouvelles pratiques. (Fassin et al., 2013: 349)

Cette affirmation prend un sens plus profond dès lors qu' elle se trouve liée à l'enquête ethnographique comme le fait D. Fassin. C'est la preuve par l'enquête ethnographique qui nous confirme la "résistance" à la totalisation de l'État (Fassin et al., 2013: 354) et par conséquent dessine une tâche spécifique pour l'anthropologue sur le terrain: le souci de se concentrer sur les pratiques concrètes, les institutions, les professions, les agents..., etc. C'est dans et par cet “ensemble composite" (Fassin et al., 2013: 350) que prennent vie les politiques publiques et l'État. D'où il est possible d'affirmer qu'il n'y a pas une raison d'État, mais plusieurs raisons croi- 
sées dans les pratiques quotidiennes. Rappelons concrètement que l'Introduction, appelée “Au Cœur de l'État”, nous montre l'importance de l'analyse des pratiques quotidiennes des institutions et leurs effets sociaux pour les études de politiques sociales. C' est un effort considérable de bâtir une approche ethnographique pour l'étude de l'État et, dans un sens plus général, de poser des jalons concrets pour une “désontologisation” de l'État. C'est-à-dire, montrer comment l'État n'est pas une entité monolithique, mais qu'il serait plutôt une figuration d'une unité normative qui en réalité est objet d'interprétation et de l'action des intervenants concrets dans chaque institution et que c'est, par leurs actions, qu'il s'inscrit concrètement dans la vie quotidienne.

Dans la perspective soutenue par “Juger, réprimer et accompagner”, l'expression “plonger au cœur de l'État” est avant tout une démarche ethnographique qui prend en compte "(...) les valeurs et les affects qui traversent les politiques des gouvernants et les pratiques des agents" (Fassin et al., 2013: 13). La clé de voûte de cette démarche est sans doute l'investissement ethnographique présent dès le début de l'argumentation de l'Introduction et une perspective d'observation tournée vers les sujets en action. En effet, l'Introduction commence avec une présentation d'extraits de travail de terrain, de véritables flashs ethnographiques qui permettent de poser la question de la "désontologisation" dans des termes concrets. Entre la loi, la police, les pratiques juridiques et les services de soutien aux victimes, il n'y a pas de politiques directement en action. Chacune de ces institutions prend vie à partir d'un champ où elles se construisent concrètement dans l'action, par les pratiques des agents concrets. Par exemple, d'une part, en matière de police et de justice il nous est dit qu' elles "trouvent leur réalisation la plus accomplie dans l'administration des populations et des territoires marginaux. D'autre part, même au centre de l'appareil étatique, les institutions peuvent déroger aux lois qu'il édicte et aux normes qu'il promeut." (Fassin et al., 2013: 15)

Le mot "déroger" est fondamental ici pour souligner non seulement un manque vis-à-vis des normes édictées par l'État, mais surtout l'importance des sujets comme des éléments fondamentaux de la construction des politiques publiques. Il nous ouvre une voie d'analyse qui prend les sujets comme des agents médiateurs entre les politiques et les pratiques quotidiennes par lesquelles l'État prend vie. L'auteur nous invite alors à prendre en compte l'action des sujets, qu'il affirme ne pas être "déterminée" par un cadre normatif, qui est plutôt une référence d'action, en quelque sorte le ressort de l'action. Ainsi, pour l'ethnographie de l'État, il 
faudra s'en tenir "plutôt qu'à la 'personne morale' dématérialisée et à cette 'administration' impartiale dont parlent les dictionnaires. Nous portons notre attention sur les personnes physiques qui le constituent et les raisons historiques que le traversent (...)" (Fassin et al., 2013, p. 15). Dans cette acception, l'État serait un produit du temps et des luttes sociales et non pas un aboutissement, essentialisé et permanent, il serait de l'ordre du mouvant. Il s'agit d'un degré de plus vers l'approche ethnographique suggérée encore une fois par l'affirmation: “L'État se déploie à travers l'ensemble de ces professionnels qui, simultanément, appliquent et produisent l'action publique." (Fassin et al., 2013:16)

C'est en ces termes qu'il nous est proposé une approche "transversale", qui nous semble “(...) d'autant plus justifiée que l'État auquel nous nous intéressons qui gouverne les populations, laissant à ses agents une importante marge de manœuvre dans la gestion concrète des situations et des individus dans leurs rapports de dépendance, voire de domination." (Fassin et al., 2013: 16) Il s'agit donc d'une démarche dont la toute première caractéristique est d'être ethnographique et par là, se donnant, à juste titre, comme tâche de se concentrer sur les institutions, où les agents sont en action et où ils objectivent par leurs pratiques l'État et les politiques sociales. L'approche proposée dans cette Introduction pour l'étude de l'État est donc avant tout une direction pour la recherche qui nous pousse à sortir du domaine normatif ou du simple "devoir être", et nous concentrer dans le "comment" et "par qui” l'État prend forme quotidiennement. Cependant, si nous avons franchi un pas vers les institutions et les pratiques, il reste encore un point à décortiquer ici, précisément celui de préciser le rôle de médiateur d'une institution, d'une profession ou des individus, ceux qui font usage d'une "marge de manœuvre" (Fassin et al., 2013: 17) et c'est bien de cette question que traite le prochain mouvement de cette exégèse.

\section{Vers les sujets}

À partir des considérations qui nous donnent les fondamentaux de la désontologisation de l'État, tout particulièrement dans le sens du "dégorgement" et plus directement à propos des "marges de manœuvre” des individus, l'Introduction du livre soutient que:

On peut aller plus loin. Il ne s'agit pas seulement d'affirmer que les agents ont une marge d'interprétation et une liberté de manœuvre par rapport aux injonctions 
des textes ministériels et parlementaires qui définissent les modalités de 'tactiques' qui, selon Michel de Certeau, impliquent que les agents , n'ayant pas la maitrise des règles du jeu, ne peuvent que les détourner, alors que les 'stratégies' s'inscrivent dans un espace où ils disposent d'une certaine autonomie pour les définir. En réalité, souvent, par excès de zèle ou par conviction, les agents développent les politiques bien au-delà de ce qui leur est demandé: en ce sens, ils ne se contentent pas d'appliquer la politique de l'État, ils la font; ils sont l'État. (Fassin et al., 2013: 17)

Cet extrait de l'ouvrage fait appel aux idées séminales de M. de Certeau (2004) qui sont fort adéquates pour situer la place des sujets dans son analyse. Cependant, puisque l'auteur fait plusieurs références aux travaux de P. Bourdieu, il serait intéressant de rappeler les différences entre l'approche bourdieusienne et les propositions de M. de Certeau pour avancer ma propre réflexion. Je retiendrai dans les limites de mon propos ici deux points de cette critique: 1) l'effort de mettre en évidence que les "acteurs" sont actifs dans un sens fort qui est celui de leur inventivité, leurs "manières de faire"; 2) l'irréductibilité des pratiques quotidiennes. Au pair habitus-champ bourdieusien, qui est une contribution théorique très importante dans l'histoire des sciences sociales, nous avons chez M. de Certeau le complexe pair stratégies-tactiques. Il n'y a pas d'équivalences entre ces deux paires analytiques, puisque les contributions de $\mathrm{M}$. de Certeau nous font valoir les sujets en action: les stratégies impliquent un calcul des rapports de force à partir desquels se définit un lien propre (une intériorité) et une extériorité. Ce qui donne un sens concret à l'idée du "dégorgement", mais peut-être moins pour ce qui est de la "marge de manœuvre", puisque les sujets sont liés à des "manières de faire", non limitées d'avance, à des possibilités de jeu qui échappent au contrôle par leur propre initiative.

Je suis d'accord avec l'argumentation de l'Introduction et son appel aux questions posées par M. de Certeau, qui sont très en phase avec les besoins de la recherche ethnographique, au sens de l'observation des acteurs en action et, surtout, de l'irréductibilité des pratiques observées sur le terrain. À ce stade de l'argumentation, il y a un dépassement du schéma bourdieusien, puisque M. de Certeau fait appel à l'inventivité des sujets qui échappent au contrôle, à leurs "manières de faire" concrètement mises en œuvre, et à la création d'un espace “intérieur” ou encore au jeu qui peut subvertir les normes et les directives d'action des institutions. Ce jeu ne se limite pas à l'incorporation" d'un habitus ou d'une "socialisation" qui sont, par 
ailleurs des éléments dont les sujets disposent pour se mettre en action.

Il me parait nécessaire de s'affranchir des limites de l'habitus et poser plus radicalement la question de l'inventivité des sujets. Cependant, dans le texte, nous pouvons identifier une certaine, disons, "oscillation” entre les deux approches. En fait, sans perdre de vue le fil de l'inventivité, le texte revient à l'approche bourdieusienne quand il affirme: "le point de rencontre des politiques et des pratiques, l'interface entre ce qui se joue dans l'espace public et dans l'habitus professionnel." (Fassin et al., 2013: 19). Ou encore dans les passages suivants:

D'une part, les institutions voient leur action encadrée par une législation, une allocation de ressources et une organisation des moyens qui en déterminent, au moins en partie, les modalités: les logiques définies dans la loi organique relative aux finances, les outils statistiques d'évaluation de la performance, l'évolution des affectations de personnels, la transformation des structures opèrent comme autant de contraintes qui circonscrivent les possibles, mais génèrent aussi des innovations. Mais, d'autre part, les agents travaillent également en référence à un certain éthos de leur profession, à une formation qu'ils ont reçue, à une idée qu'ils se font de leur action et à une routine qui s'impose à eux: les principes d'ordre ou de justice, les valeurs de bien commun et de service public, l'attention aux réalités sociales ou psychiques ou bien l'ignorance des unes et des autres, tous produits de l'habitus de leur métier, influencent la manière dont ils vont répondre aux injonctions étatiques et se comporter vis-à-vis du public. (Fassin et al., 2013: 19)

Ou encore:

Ce n'est donc pas seulement l'État qui dicte une politique aux agents, et sont aussi les agents qui font la politique de l'État, en étant plus ou moins contraints par le cadre de leur métier et de leurs ressources, en prenant plus ou mois d'initiatives par rapport à la règlementation qui s'impose à eux et, finalement, en politisant, au sens fort, leur action, c'est-à-dire en lui donnant une signification politique. (Fassin et al., 2013: 18)

Il serait intéressant ici de rappeler la critique des approches individualistes et la façon dont elle est construite dans l'Introduction:

Depuis deux ou trois décennies, l'étude des institutions a bénéficié d'un important regain d'intérêt dans les sciences sociales, en théorie politique et en histoire économique. Alors que les approches individualistes, qui ont longtemps dominé ces 
disciplines, mettent en avant les choix d'acteurs supposés rationnels et décrivent des agrégations de comportements nécessairement vouées à une certaine volatilité, les approches dites institutionnalistes privilégient la compréhension de ce qui fait que, comme l'écrivent Élisabeth Clements et James Cook, "les institutions durent” en s'appuyant des organisations sociales. (Fassin et al., 2013: 20)

Ainsi, le développement qui nous est proposé dans cette Introduction va dans le sens de l'adoption des conceptions de M. de Certeau (2004). Mais il semble que nous pouvons faire un mouvement de plus, en ce qui concerne l'inventivité des sujets. En effet, il y a des références plutôt sur l'habitus tout au long du texte, encore que dans son récit ethnographique, les empreintes de M. de Certeau sont nettement visibles, particulièrement à travers le traitement de l'irréductibilité des pratiques quotidiennes. Je crois qu'il faut faire ce pas décisif pour accomplir pleinement l'objectif de la désontologisation de l'État.

En résumé, les remarques théoriques proposées dans l'Introduction forment un ensemble qui soutient parfaitement les propos de faire la critique de l'État comme une totalité achevée et nous propose un chemin pour redresser le focus de l'enquête anthropologique vers les sujets qui, dans leurs pratiques quotidiennes, font l'État. C'est sans doute, un pas décisif pour l'ethnographie que celui de faire l'observation et la description des agents comme de véritables médiateurs, au sens d'observer et de décrire les opérations par lesquelles les politiques publiques acquièrent une substance concrète. C'est par et avec ces agents que les lois, les règlements administratifs, l'organisation du travail, etc. donnent une vie concrète aux politiques sociales. Mes observations sont une espèce de dièse théorique pour montrer que la voie ouverte par le texte commenté porte des éléments pour la discussion sur le sujet tel qu' envisagée ici. Il s'agit donc de quelques points de repère pour suivre un fil conducteur déjà proposé.

Cependant, le “mot de passe”, pour avancer dans la réflexion, je l'ai trouvé dans une brève référence à J. Butler dons les réflexions étaient présentes dans mes analyses antérieures (Rifiotis, 2014a, 2015). Reprenons donc les termes par lesquels l'œuvre de J. Butler est évoqué:

Du reste, on peut aller plus loin et le fait remarquable n'est pas seulement que les institutions durent, mais que la société se produit et se reproduit à travers elles: les agents ne se comportent pas comme des électrons libres, mais se trouvent pris dans des réseaux de signification et d'action inscrits dans les institutions. La fonction de ces dernières a du reste une double valence: d'un côté, contrainte qui 
impose des limites à la liberté des agents; de l'autre, dispositif qui constitue les formes du vivre ensemble. Dans les termes de Judith Butler, nous sommes à la fois assujettis et subjectifs par les institutions, et notamment par la plus visible d'entre elles: l'État. (Butler, 2009 : 20-21)

Retenons de cet exergue l'affirmation de la "double valence" et la double condition d'assujetti et de sujet. Pour aller plus loin dans ce propos, il nous faudra poser plus radicalement la question de quel sujet parlons-nous et d'en tirer les conséquences théoriques, politiques et éthiques au sens latin du terme (subjectus) et de sa version moderne d'agent.

\section{Quel sujet?}

Tout d'abord, rappelons-nous que la question du sujet dans les études de la morale n'est pas une nouveauté, comme il est possible de le constater déjà dans le livre publié sous la direction de S. Howell, intitulé "The Ethnography of Moralities" (1997), dans lequel elle affirme:

One question that arises in the study of moralities is whether moral codes and values must necessarily involve individual self-awareness in some form or another, and whether moral reasoning is performed by every individual regardless of social-cultural conditions and ideological constructs. (Howell, 1997: 14)

Le renouveau, si je peux m'exprimer ainsi, tel que je l'envisage, serait seulement un pas de plus vers une théorie du sujet comme centre analytique. Mais de quel sujet est-il question? Cette précision me parait fondamentale pour bien comprendre les enjeux qui en découlent. Ainsi, pour essayer de répondre à la question de quel sujet s'agit-il, il me semble qu'il faudra revenir sur un ensemble de références que je ne peux présenter ici que d'une manière synthétique compte tenu de la complexité du propos et des mes propres limites. Commençons par dire clairement que mes propos ne vont pas dans la direction d'un "retour du sujet" au sens plat de l'individualisme comme l'affirme aussi Eduardo Viveiros de Castro (s/d).

Le sujet, à mon sens, ne serait donc pas une identité, ni une totalité, ni un individu ou acteur avec un background, ni une subjectivité donnée, mais un processus qui a lieu par et dans l'action des agents. Il s'agit - dans les termes de l'Introduction - de la "production de sujets" (Fassin et al., 2013: 24). Cette expression je la comprends comme un résultat des considérations autour de ce texte; elle fait appel à l'”articulation" entre assujettissements et subjectivation et qui devient centrale pour 
le travail ethnographique:

L'articulation de ces deux concepts, telles que nous la proposons à travers nos ethnographies des institutions, permet de lier les deux grandes approches des questions morales, d'inspiration kantienne ou aristotélicienne, autrement dit l'éthique du devoir et l'éthique de la vertu, dans les sciences sociales depuis un siècle. Selon la première, toute société se caractérise, à un moment donné, par un ensemble de normes et de valeurs qui définit une sorte de code moral auquel les individus doivent se soumettre soit par obligation d'accomplir leur devoir, soit par désir de bien faire. Selon la seconde, tout individu peut développer, indépendamment des règles qui sont collectivement imposées, des pratiques vertueuses vis-à-vis de lui-même et vis-à-vis des autres. Le premier approche souligne la contrainte, la seconde la liberté. À la différence des codes, les économies morales permettent cependant de saisir des changements dans le temps et les appropriations par les agents: les normes et les valeurs ne leur sont pas simplement imposées et, de plus, elles s'associent à des émotions et des sentiments. À la différence des vertus, les subjectivités morales ne se réfèrent pas seulement aux pratiques orientées vers la recherche du bien, mais intègrent toutes les formes de pratiques ayant un contenu moral, quelle que soit sa valence. (Fassin et al., 2013: 24)

\section{Et un peu plus loin:}

Économies morales et subjectivités morales trouvent en quelque sorte leur articulation dans l'activité quotidienne des institutions par le jeu des valeurs et des affects qui se cristallisent autour des questions de société et des réponses qui leur sont apportées dans des institutions concrètes: pour le policier, le magistrat, le conseiller d'insertion et probation, l'agent orienteur de la mission locale, ce sont, respectivement, l'insécurité qui s'incarne dans l'adolescent de la cité, la suspicion dans le demandeur d'asile, la dangerosité dans le détenu libérable, le chômage dans le jeune discriminé - chacun de ces cas se posant aux professionnels à travers des évaluations et des émotions, des jugements sur ce qu'est un vrai réfugié ou un bon prisonnier et des indignations à l'encontre d'un manque de respect ou d'un défaut de justice. (Fassin et al., 2013: 25)

Mais s'agit-il de "marge de manœuvre" ou de tactiques? La première option renvoie à "marge d'interprétation" et à "autonomie". Tandis que la seconde prône plutôt l'invention des sujets, ce qui n'est pas tout à fait équivalent. Au lieu d'avancer dans un choix, à ce stade de la réflexion, il sera plus utile d'approfondir les réfé- 
rences de base et revenir aux travaux de M. Foucault compte-tenu des dialogues entre son œuvre et celle de J. Butler citées dans le texte. Mais pour synthétiser cette révision je m'attarderai plus particulièrement sur la façon par laquelle $\mathrm{M}$. Foucault envisage la question de la morale et le sujet.

Chez M. Foucault le sujet et la morale sont analysés à partir de trois perspectives distinctes et entrecroisées (1984: 34):

1) La morale comme un code, des valeurs

2) La morale comme pratique, les comportements d'observance ou de résistance

3) la morale comme une évaluation et un choix, la morale en acte à partir des évaluations du sujet, le développement du "travail éthique" sur soi-même

À partir de cette triple approche, l'analyse de la morale doit faire face à une nouvelle exigence: le "travail éthique" sur soi-même, ou dans les termes de M. Foucault: “(...) L'action morale est indissociable de ces formes d'activité sur soi (...)” (1984: 40). Or, c'est bien sûr vers le sujet en action, cette automodélisation, que se tourne le regard analytique, c'est dans la gravité de son action que la morale prend du sens.

Une manière complémentaire de penser la relation entre sujet et morale c'est de l'aborder par le biais de l'assujettissement. Dans les travaux de M. Foucault, nous trouvons une formulation assez complexe et difficile à saisir, dont la formulation de J.Butler me parait plus claire, tout au moins quant à la relation entre sujet et norme. Dans son livre "Dar cuenta de sí mismo. Violencia ética y responsabilidade" ("Rendre compte de soi-même. Violence éthique et responsabilité") (2009), elle nous aide à problématiser cette question dans les termes suivants:

La norme ne produit pas le sujet comme son effet nécessaire ni le sujet ne peut non plus ignorer la norme qui établit sa réflexivité. (...) Si dans cette lutte il y a de l'agentivité ou de la liberté, elle se produit dans un cadre à la fois porteur et limitant de coercition. L'éthique de l'agentivité n'est jamais complètement déterminée ni libre. (Butler, 2009: 33)

Il y a là une question cruciale: le rapport présupposé entre la norme et le sujet qu'il faut problématiser d'une manière radicale. Les relations entre normes et sujet ne sont pas de détermination ou incorporation, elles sont en même temps contraignantes et aussi les conditions de l'émergence du sujet. Dans un sens plus large, nous pourrions dire que l'assujettissement n'est pas seulement une capture, mais 
qu'il produit une subjectivité, condition de l'émergence du sujet. Donc, paradoxalement, assujettissement et résistance sont un couple inséparable.

Dans ce chantier théorique, il y aurait le travail de S. Ortner et les "jeux sérieux" (“serious games") et sa conception du sujet (2007a, 2007b). Sans en faire une révision exhaustive, je crois pertinent de rappeler les trois propositions de S. Ortner quant à sa contribution au débat sur la place du sujet dans la théorie sociale:

1) la question de l'"unité d'action": acteurs individuels (individus ou groupes sociaux). L'individu ou le collectif comme entité singulière.

2) la question temporale de l'action: pour Bourdieu l'action est ad hoc tandis que pour Ortner il s'agit de projets construits et mis en action par les sujets.

3) la question des types d'action: contre l'idée de mise en scène de T. Parsons, l'approche pragmatique met en avant les choix, la prise de décision, le calcul ou les stratégies.

Les trois points que S. Ortner développe tout au long de ses œuvres sont en strictes relations avec l'idée d'agentivité et les buts que les sujets se donnent au cours de leurs actions. Ce qui me parait rejoindre les propos sur le sujet comme un agent qui était déjà présent dans les propos de M. Foucault ou de J. Butler, et bien sûr chez M. de Certeau.

Pour en revenir à la question de base, je dirais que le sujet n'est pas un individu doté de conscience et autonomie ni un acteur au sens dramaturgique du terme comme "rôle social", ni une personne au sens anthropologique (voir M. Leenhardt (1971) et plus récemment M. Strathern (2006)). Il ne s'agit pas non plus d'une identité ou d'une subjectivité donnée et fixe. Le sujet contemporain, comme l'a bien montré S.Hall (2000), est fragmenté et morcelé.

$\mathrm{Au}$ fond, nous rejoignons encore une fois la proposition de M. de Certeau selon laquelle chaque individualité est un lieu où se croisent des pluralités, où nous pouvons nous rendre compte que "le quotidien s'invente avec mille manières de braconner”. (Certeau, 2004: XXXVI) Nous avons, jusqu'à présent, essayé de circonscrire cette pluralité à partir d'études ethnographiques de longue durée dans les institutions que reçoivent des "jeunes contrevenants" ou "jeunes en conflit avec la loi” à Santa Catarina dans le sud du Brésil (Rifiotis et al., 2016). Après de longues années de travail de terrain, nous avons cherché à interpréter la pluralité identitaire que nous avons observée chez ces jeunes. La question que nous nous sommes 
posée, c'est exactement celle qui m’occupe présentement l'esprit sur le plan théorique: de quel sujet s'agit-il? Pour les mouvements de défense des droits des jeunes, n'étaient-ils que des "victimes" ou des "jeunes en conflit avec la loi” tandis que pour ceux qui soutiennent la baisse de l'âge de la majorité pénale, ils ne sont que des "agresseurs" ou tout simplement des “criminels". Ce qui fait le débat selon des enjeux théoriques, mais aussi politiques et éthiques.

Sur le terrain, nous nous sommes rendus compte qu'un même jeune faisait des récits sur lui-même fort différents, parfois comme "victime", parfois comme "mauvaise personne", ou encore comme "sujet homme" (c'est-à-dire éthique, celui qui n'était pas un délateur et qui respectait ses "amis" et "alliés", ou simplement comme de "vida loka" au sens de celui qui vit dans le danger, dans l'adrénaline, qui prend des risques et aime la déviance. En analysant les ethnographies accumulées au fil des ans, nous sommes parvenus à la compréhension qu'à propos d'un même évènement (et son autoqualification) le récit de chaque jeune prenait de tournures particulières, nettement distinctes, selon le contexte d'énonciation et/ou de son interlocuteur. Pour essayer de rendre compte du caractère multiple de se représenter ces jeunes, nous avons utilisé l'expression "sujet-composite" qui nous a permis de mettre en évidence le caractère relationnel et contingent de ces représentations. C'était un tout premier tâtonnement de la pluralité du sujet, pas conclusif, mais une voie qui me parait prometteuse (Rifiotis et al., 2016).

Si la notion bourdiesienne de "champs" et "habitus" chez P. Bourdieu, ainsi que les efforts théoriques de A. Giddens et M. Sahlins, ont été un pas important pour la théorie anthropologique contemporaine pour affranchir la dichotomie individu/ société, il demeure un autre seuil avec la notion de sujet en devenir. Dans une telle perspective, je crois qu'il s'agit de penser le sujet de l'action plutôt comme un résultat et non pas comme un point de départ de l'action. Le sujet est à la fois un agent et un réseau, c'est-à-dire la série de connexions qui ont lieu au cours de l'action. Dans ce sens, il sera très prometteur de penser le sujet - au cours de l'action et en relation - avec le travail entrepris sur soi-même et dont les résultats portent une certaine imprévisibilité. En fait, inspiré particulièrement par les travaux de Michel Callon, Bruno Latour, plus récemment, je me suis tourné vers une approche plus performative du social, mais c' est une voie à peine esquissée. La lecture du dossier "Un tournant performatif ? Retour sur ce que 'font' les mots et les choses" publié dans la Revue Réseaux en 2010 fait état de travaux intéressants dans cette approche. Pour résumer, il me paraît que s'ouvre un voie puissante pour l'analyse anthropo- 
logique et concrète dans la "désontologisation” de l'État.

\section{Quelques considérations finales}

Parvenu au terme de ces remarques préliminaires, je voudrais rapidement insister sur la notion de sujet défendu ici et quelques-uns de ses effets pour les études des économies morales. Rapidement et sans prétention autre que celle de soulever quelques points qui en découlent, je retiendrai provisoirement les points suivants:

Tout d'abord, il me parait évident qu'il faudra poursuivre les études sur le sujet. Revenir sur les classiques en premier M. Mauss (1974) et M. Leenhardt (1971), et plus récemment L. Dumont (1983). Mais aussi sur les contributions plus actuelles de J. Butler (2009), M. Strathern (2006), M. Agier (2012) et E. Balibar (2012), par exemple. Il y a une longue histoire de la notion de sujet a récupérer et à identifier dans les recherches ethnographiques.

D’une manière générale, rappelons ici que le sujet est un braconnier. Il ne sera donc pas question de considérer exclusivement sa profession ou les institutions dans lesquelles il agit ou de sa formation, incorporation ou déontologie. Elles sont des références autour desquelles gravitent la formation des sujets et des modes de subjectivation. Mais ce mode de subjectivation se croise avec l'âge, le genre, l'ethnie, etc., et surtout avec le cadre relationnel dans lequel chaque action précise s'inscrit, c'està-dire avec le caractère contingent de l'action. Ou comme l'avait déjà souligné $\mathrm{M}$. de Certeau: c'est la relation qui détermine les termes et non pas l'inverse, dans cette phrase lapidaire: "chaque individualité est le lieu où joue une pluralité incohérente (et souvent contradictoire) de ses déterminations relationnelles” (Certeau, 2004: 38).

Un des effets pratiques de ces débats c'est le centre des politiques publiques. Par exemple, si nous gardons comme point de départ analytique la notion d'habitus, produire de changement dans une institution sera avant tout une question de changement de culture ou un changement organisationnel. Cependant, si nous considérons l'inventivité ou l'agentivité des sujets et son incomplétude, comme dans chaque action, le sujet crée et recrée son existence et ses rapports avec autrui, notre champ de recherche et en conséquence d'action, s'élargit d'une manière jusqu'à maintenant inattendue. Notre manière de mener la recherche ethnographique (choisir ce qui doit être observé et décrit) devrait-elle changer radicalement?

Une dernière remarque qui me parait tout à fait dans la démarche de "Juger, réprimer et accompagner": dans un cadre de déclin de l'État social en faveur de l'État pénal et Liberal, comment penser la place des citoyens comme protagonistes 
sociaux? Ou encore: comment agir analytiquement, politiquement et éthiquement entre la solidarité, la victimisation et l'agence des sujets? (Rifiotis, 2014a).

Recebido: 21/03/2018

Aprovado: 06/09/2018

Theophilos Rifiotis é professor Titular do Departamento de Antropologia da Universidade Federal de Santa Catarina. É coordenador do Laboratório de Estudos das Violências (LEVIS), do Programa de Pós-Graduação em Antropologia Social, UFSC.Contato: rifiotis@gmail.com

\section{Notes}

1. Le texte de cette présentation intitulé "Between theory, ethics and morals: Rethinking the place of a 'moral economy' in the social agenda for the production of justice" a été soumis à la revue Anthropological Theory pour la publication.

2. Cette démarche m'a été indiquée par D. Fassin quand je lui ai exposé l'idée générale de cette réflexion. Je lui suis reconnaissant de cette indication et de la possibilité d'approfondir mes connaissances dans ce domaine.

3. Il faut souligner que "Juger, réprimer et accompagner" est un ouvrage collectif, sans signature de chapitres, il s'agit de "rédacteurs", comme mentionné dans une courte note de l'édition, dont la liste nominative permet d'identifier les "rédacteurs" de chaque chapitre. En l'occurrence, l'Introduction qui fait objet de cet essai a comme "rédacteur" D. Fassin. De telle sorte que je préfère appeler ici "auteur" ou "rédacteur" pour garder l'esprit collectif de l'ouvrage, encore qu'il soit claire que la rédaction de l'Introduction, ainsi que du chapitre 4 appelé "Maintien de l'ordre” et les Conclusions sont de la responsabilité de Didier Fassin.

4. "Problématisation ne veut pas dire représentation d'un objet préexistant, ni non plus création par le discours d'un objet qui n' existe pas. C'est l'ensemble des pratiques discursives ou non discursives qui fait entrer quelque chose dans le jeu du vrai et du faux et le constitue comme objet pour la pensée (que se soit sur la forme d'une réflexion morale, de cela connaissance scientifique, de l'analyse politique, etc.).” (Foucault, 1984: 18)

5. En plus, les demandes pour droits et plus particulièrement pour la criminalisation sont porteurs d'une rationalité pénale, c'est-à-dire d'un mélange, un véritable “carambolage”, entre deux niveaux de normes distinctes entre normes de comportements et normes de sanction (Pires, 2004). 
6. Certes, le pouvoir discrétionnaire est intrinsèque à ses métiers et à ses institutions, mais il en reste le fait que par les choix que se font les agents concrètes dans ces places soit produite la justice par des manières fort différentes. Ce qui peut être d'un vecteur d'asymétrie de genre et reproduction de valeurs pas nécessairement compatible avec les attentes des mouvements sociaux, l'esprit de la loi" et des politiques de protection des femmes. Cette "liberté" est un des enjeux de la question du sujet et de mon propos dans cet essai.

\section{Bibliographie}

AGIER, Michel. 2012. Penser le sujet, observer la frontière. Le décentrement de l'anthropologie. Paris: L'Homme, (203-204):51-75.

BALIBAR, Étienne. 2012. Citoyen sujet et autres essais d'anthropologie philosophique. Paris: PUF.

BENJAMIN, Walter. 1978 (ed. orig. 1921). "Critique of violence”. In: BENJAMIN, W. Reflections. Essaye, Aphorisms, AutobiograficalWrittings. New York, London: ABJ Book. pp. 277-300.

BOURDELAIS, Patrice; FASSIN, Didier. 2005. Les constructions de l'intolérable. Paris: La Découverte.

BRAGAGNOLO, Regina Ingrid; LAGO, Mara; RIFIOTIS, Theophilos. 2015. "Estudo dos modos de produção de justiça da Lei Maria da Penha em Santa Catarina”. Revista Estudos Feministas, (23): 601-617.

BUTLER, Judith. 2009 (ed. orig. 2005). Dar cuenta de sí mismo.Violencia ética y responsabilidade. Buenos Aires: Amorrortu.

CALLON, Michel; LATOUR, Bruno. 2006. “Le grand Léviathan s'apprivoise-t-il?” In: AKRICH, Madeleine; CALLON, Michel; LATOUR, Bruno. Sociologie de la traduction. Textes fondateurs. Paris: Presses de Mines. pp.11-32.

CARDOSO DE OLIVEIRA, Luís. 2008. “Existe violência sem agressão moral?” Revista Brasileira de Ciências Sociais, 23(67):135-146.

CERTEAU, Michel De. 2004. (orig. 1990). A invenção do cotidiano.Volume 1. Artes de Fazer. Petrópolis: Editora Vozes.

CLASTRES, Pierre. 1988. A sociedade contra o Estado. Rio de Janeiro: Francisco Alves.

DOSSIER “Un tournant performatif? Retour sur ce que 'font' les mots et les choses”. 2010. Réseaux, 5(163).

DUMONT, Louis. 1983. Essais sur l'individualisme. Une perspective anthropologique sur l'idéologie moderne. Paris: Le Seuil.

FASSIN, Didier. 2008. "Beyond good and evil? Questioning the anthropological discomfort with morals”. Anthropological Theory, 8 (4):333-344.

FASSIN, Didier. 2009. "Les économies morales revisitées”. Annales. Histoire, Sciences Sociales, (6):1237-1266.

FASSIN, Didier; RECHTMAN, Richard. 2007. L'empire du traumatisme. Enquête sur la condition de victime. Paris: Flamarion.

FASSIN, Didier; BOUAGGA, Yasmine; COUTANT, Isabelle; EIDELIMAN, Jean-Sébastien; FERNANDEZ, Fabrice; FISCHER, Nicolas; KOBELINSKY, Carolina; MAKAREMI, Chowra; 
MAZOUZ, Sarah; ROUX, Sébastien. 2013. Juger, réprimer, accompagner: essai sur la morale de l'État. Paris: Éditions du Seuil.

FOUCAULT, Michel. 1984. L'usage des plaisirs. Histoire de la sexualité II. Paris: Gallimard.

GLUCKMAN, Max. 1963. Order and rebellion in tribal Africa. London: Cohen \& West.

HALL, Stuart. 2000 (ed. orig. 1992). A identidade cultural na pós-modernidade. Rio de Janeiro: DP\&A.

HOWELL, Signe. 1997. "Introduction”. In: HOWELL, S. (org.) The Ethnography of Moralities. London: Routledge. pp. 1-22.

KATZ, Jack. 1988. Seductions of crime. Moral and sensual attractions in doing evil. New York: Basic Books.

LEENHARDT, Maurice. 1971 (ed. orig. 1947). Do Kamo: la personne et le mythe dans le monde mélanésien. Paris: Gallimard.

MAUSS, Marcel. 1974 (ed. orig. 1938) "Uma categoria do espírito humano: a noção de pessoa, a noção do ‘eu'”. In: MAUSS, M. Sociologia e Antropologia. vol. 1. São Paulo: EPU/EDUSP. pp. 207241.

ORTNER, Sherry. 2007a. "Poder e Projetos: reflexões sobre a agência”. IN: GROSSI, Miriam Pilar; ECKERT, Cornélia ; FRY, Peter. (org.). Conferências e Diálogos: saberes e práticas antropológicas. Blumenau: Nova Letra. pp. 45-80.

ORTNER, Sherry. 2007b. "Subjetividade e crítica cultural”. Horizontes Antropológicos. UFRGS, 13(28): 375-405.

PIRES, Alvaro. 2004. “A racionalidade penal moderna, o público e os Direitos Humanos”. Novos Estudos, (68): 39-60.

RIFIOTIS, Theophilos. 1997. "Nos campos da violência: diferença e positividade”. Antropologia em Primeira Mão. Florianópolis: Programa de Pós-graduação em Antropologia Social, Universidade Federal de Santa Catarina, (19):1-30.

RIFIOTIS, Theophilos. 1998. "Dilemas éticos no campo da violência”. Revista Educação \& Comunicação. Escola de Comunicação. São Paulo: Universidade de São Paulo. (13):26-32.

RIFIOTIS, Theophilos. 1999. "Violência policial na imprensa de São Paulo. O leitor-modelo no caso da Polícia Militar na Favela Naval (Diadema)”. Revista São Paulo em Perspectiva. São Paulo: Fundação Seade , 13(2):28-41.

RIFIOTIS, Theophilos. 2004. "As delegacias especiais de proteção à mulher no Brasil e a judicialização dos conflitos conjugais”. Revista Sociedade e Estado. Brasilia: UNB, 19(1): 85-119.

RIFIOTIS, Theophilos. 2006. "Alice do outro lado do espelho: revisitando as matrizes do campo das violências e dos conflitos sociais”. Revista de Ciências Sociais. Fortaleza: Universidade Federal do Ceará. 37(2):27-33.

RIFIOTIS, Theophilos. 2007a. "Ancestrality and age conflicts in Back-African societies." In: AGUILAR, M. I. (org.) Rethinking Age in Africa: Colonial, Post-Colonial, and Contemporary Interpretations of Cultural Representations. Trenton: Africa World Press. pp.199-225.

RIFIOTIS, T. 2007b. "Sujeito de direitos e direitos do sujeito”. In: SILVEIRA, R.M.G. et al. Educação em Direitos Humanos: fundamentos teórico-metodológicos. João Pessoa: Editora Universitária. pp. 


\section{1-244.}

RIFIOTIS, Theophilos. 2008a. "Violência e poder: avesso do avesso?" In: NOBRE, R. F. O poder no pensamento social: dissonâncias do mesmo tema. Belo Horizonte: Editora UFMG. pp.153-173.

RIFIOTIS, Theophilos. 2008b. “Judiciarização das relações sociais e estratégias de reconhecimento: repensando a 'violência conjugal' e a 'violência intrafamiliar'”. Revista Katálisys. Florianópolis, Universidade Federal de Santa Catarina, 11(2):225-236.

RIFIOTIS, Theophilos. 2011a. "Direitos Humanos e outros direitos: aporias sobre processos de judicialização e institucionalização de movimentos sociais”. In: RIFIOTIS, Theophilos; HYRA, Tiago. (org.) Educação em Direitos Humanos. Discursos críticos e temas contemporâneos. Florianópolis: Editora da UFSC. pp. 39-58.

RIFIOTIS, Theophilos. 2011b. "Parricidio: padres e hijos en el tribunal de justicia de Florianopolis, Santa Catarina, Brasil)”. In: RIFIOTIS, T.; CASTELNUOVO, N. (org.). Antropología,Violencia y Justicia. Repensando matrices de La sociabilidad contemporánea en el campo del género y de la família. Buenos Aires: Editorial Antropofagia. pp.91-124.

RIFIOTIS, Theophilos. 2014a. "Entre teoria, estética e moral: repensando os lugares da antropologia na agenda social da produção de justiça”. Antropologia em Primeira Mão, PPGAS/UFSC, (141):1-23.

RIFIOTIS, Theophilos. 2014b. "Judicialização dos direitos humanos, lutas por reconhecimento e políticas públicas no Brasil: configurações de sujeito”. Revista de Antropologia. São Paulo, USP, 57(1):119-149.

RIFIOTIS, Theophilos. 2015. "Violência, Justiça e Direitos Humanos: reflexões sobre a judicialização das relações sociais no campo da 'violência de gênero'”. Cadernos Pagu. Campinas: Universidade de Campinas, (45):261-295.

RIFIOTIS, Theophilos; VIERA, Danielli; DASSI, Tatiana. 2016. “Judicialização das relações sociais e configurações de sujeito entre jovens cumprindo medidas socioeducativas em Santa Catarina”. Anuário Antropológico. Brasília, UnB, 41(1):35-55.

RIFIOTIS, Theophilos. 2017. “Judicialização das Relações Sociais”. Cadernos da Defensoria Pública do Estado de São Paulo. São Paulo, (7):26-39.

SIMMEL, George. 1992 (ed. orig. 1908). Le Conflit. Saulxures: Circé.

SOUZA LIMA, Antonio Carlos de (dir.). 2012. Antropologia e direito :temas antropológicos para estudos jurídicos. Brasília, Rio de Janeiro, Blumenau: Associação Brasileira de Antropologia, Laced, Nova Letra.

STRATHERN, Marilyn. 2006 (ed. orig. 1988). O gênero da dádiva:problemas com as mulheres e problemas com a sociedade na Melanésia. Campinas, São Paulo: Editora da UNICAMP.

ZALUAR, Alba. 1999. "Violência e Crime”. In: MICELI, Sérgio (org.). O que ler na ciência social brasileira (1970-1995). vol. 1: Antropologia. São Paulo, Sumaré, Brasília: Anpocs; Capes. pp. 13-107. 
Resumo: $\mathrm{O}$ objetivo do presente texto é apresentar um conjunto de questões teóricas, políticas e éticas que emergem de modo recorrente de trabalhos etnográficos realizados, desde 1996, pelo coletivo de pesquisa do Laboratório de Estudos das Violências (LEVIS) da Universidade Federal de Santa Catarina. Apresento, inicialmente, os elementos de base da nossa trajetória de pesquisa no campo da violência, sem qualquer pretensão de exemplaridade. Em seguida, trago uma reflexão crítica sobre estratégias teóricas e metodológicas, particularmente relacionadas à noção de "economia moral” nas nossas pesquisas. Trata-se de um exercício preliminar de sistematização focado no papel que a antropologia pode assumir na agenda social da produção da justiça no Brasil. Minha argumentação traz uma reflexão sobre a noção de economia moral e sua relevância para a análise de políticas públicas e movimentos sociais no campo da violência, justiça e direitos humanos. Especificamente, gostaria de colocar em evidência como teoria, política e ética são indissociáveis no nosso trabalho para discutir os fundamentos que guiam a nossa pesquisa em três domínios analiticamente complementares: 1) produção e caráter moral da violência; 2) lei e judicialização das relações sociais; e 3) a construção jurídica do sujeito-vítima.
Palavras-chaves: judicialização; políticas públicas; vitimização; violência de gênero; moralidades 
Abstract: The purpose of this texte is to present a set of theoretical, political, and ethical concerns that have recurrently emerged from the ethnographic research conducted since 1996 through the the Violence Research Laboratory (LEVIS) of the Federal University of Santa Catarina. After introducing the basic elements of our research trajectory in the field of violence, without any pretension to exemplarity, I will critically reflect on some specific theoretical and methodological issues, with particular reference to the importance of the notion of "moral economy" for our current research. This will be a preliminary exercise of systematization focusing on the role that anthropology can play in the social agenda concerning the production of justice in Brazil. My anthropological reflections will question the notion of moral economy and its relevance in analyzing social movements and public policies in the field of violence, justice, and human rights. Specifically, I will highlight how theory, politics, and ethics intersect in our work, in order to discuss the fundamentals that have guided our research throughout three interrelated and complementary analytical areas: 1) the production and moral character of violence; 2) law and the judicialization of social relations; and 3) the judicial construction of the subject-victim.
Keywords: judicialization; public policy; victimization; gender violence; moralities 